\title{
Can Peak-Picked ECG Be Used for Heart Rate Variability?
}

\author{
Richard E Gregg, Reza Firoozabadi, Saeed Babaeizadeh \\ Advanced Algorithm Research Center, Philips Healthcare, Andover, MA, USA
}

\begin{abstract}
Use of peak-picked ECG is generally not recommended for heart rate variability (HRV) calculations because the apparent $R$-wave peak moves around within the QRS due to the peak-picking operation. This study tests an algorithm for recovering high resolution RR intervals from peak-picked ECG.

Two databases were used for testing, simulated ECG and Holter ECG from end stage renal disease (ESRD) patients on and off hemodialysis. Twenty minute samples of single lead ECG $(n=1000)$ were generated by the Physionet HRV ECG simulator. Each ECG record had a random combination of heart rate standard deviation and additive noise. 12-lead, 48 hour Holter ECG from 51 ESRD patients was split into 20 minute segments for $H R V$ analysis. ECG was decimated to 250sps from 1000sps and then peak-picked to the final sample rate of $125 \mathrm{sps}$. High resolution $R R$ interval recovery was based on up-sampling and template matching to align beats. HRV standard deviation of RR intervals (SDNN) was calculated for each 20 min. segment. Bland-Altman analysis was used to assess bias of SDNN error across the SDNN range.

The bias of SDNN error at low SDNN values was pronounced for $R R$ intervals defined by $R$-wave peaks. $R R$ correction by template matching reduced (1) the impact of noise and (2) the SDNN error at low levels of SDNN.
\end{abstract}

\section{Introduction}

ECG sample rate reduction may be desirable when visual rhythm determination is required but precise detail is not necessary. This is the case for real-time monitoring ECG display. The waveform scrolls past and it is used for rhythm determination, not detailed analysis. Holter ECG review is similar in that long records must be reviewed in a short period of time and ECG detail is not required to determine abnormal rhythm events.

Peak-picking is a method for sample rate reduction which mostly retains the R-wave peak amplitude for waveform visualization. Relatively course sampling can make the R-wave peak appear to vary significantly because the sample times do not fall on the actual peak time. Peak picking reduces this apparent random amplitude variation by choosing a sample closer to the true peak.

The HRV guideline paper by Malik et. al. suggests that interpolation can be used to recover high resolution RR intervals from ECG with as low a sample rate as $100 \mathrm{~Hz}$ [1]. In this paper we concentrate on ECG which has gone through a non-linear operation (peak picking) negating the ability to interpolate the true timing of R-wave peaks used as the fiducial point. With low rate sampling rate, the original signal can be perfectly reconstructed as long as Shannon's sampling theorem is respected and the sample rate is twice the signal bandwidth (reference). With an antialiasing filter that can be easily realized, the sample rate must be approximately three times the signal bandwidth. Therefore, a sample rate of $125 \mathrm{sps}$ is enough for reconstructing an ECG signal with $40 \mathrm{~Hz}$ bandwidth, typical for monitoring and Holter applications. The signal can be up-sampled to get much higher time resolution. Linear reconstruction through up-sampling is spoiled however when the non-linear peak-picking operation is used to reduce the sample rate.

This paper describes an algorithm designed to recover high resolution RR intervals from low sample rate ECG, even when the ECG has been peak-picked for visual display. The RR recovery was tested for impact on time domain heart rate variability (HRV) measured on two datasets, simulated single-lead ECG and 12-lead Holter ECG recorded on patients with end-stage renal disease (ESRD) on and off of hemodialysis.

\section{Methods}

The algorithm for high resolution RR interval recovery is based on up-sampling as the first step, matching each beat to a template beat and then using the time alignment from the template match operation to correct the QRS locations. Finally, RR intervals are calculated from the corrected QRS locations.

The simulated ECG dataset was created using ECGSYN, an ECG simulator for HRV analysis testing [2]. Twenty minute samples were generated using random combinations of desired heart rate standard deviation and additive noise level $(n=1000)$. Output of the simulator included reference R-wave peak locations.

The Telemetric and Holter ECG Warehouse (THEW) end stage renal disease on hemodialysis database (E-HOL- 
12-0051-016) was the second database for testing the effect of RR interval recovery on HRV calculation [3]. The database consisted of 48 hour 12-lead ECG Holter recordings of 51 patients both on and off hemodialysis. The dataset has high resolution with a sample rate of 1000sps and $0.5 \mathrm{uV}$ amplitude resolution.

The final step in generating test ECGs was decimation from 1,000 sps to 250 sps and then peak picking. Three forms of peak picking were tested, straight downsampling, conventional peak picking and peak picking with preserved phase. The "turning-point" algorithm was used to perform the peak picking in the decimation step from 250 to $125 \mathrm{sps}$ [4]. The enhanced peak picking operation preserved the phase of the chosen point from each pair with a single bit. On reconstruction of the encoded $125 \mathrm{sps}$ signal to $250 \mathrm{sps}$, the peak location would be known and the other missing point would be set by linear interpolation.

The ECG processing to generate normal-to-normal (NN) intervals, included bandpass filtering, up-sampling to 1000sps, template matching and QRS fiducial correction from the template matching time alignment. The bandpass filtering consisted of two pole IIR high-pass and low-pass filters with 0.5 and $40 \mathrm{~Hz}$ cut-off frequencies respectively. Template matching using cross correlation was used to align each beat with a template beat to correct the QRS fiducial point. The output of the template matching operation was a normalized cross correlation value and the number of samples the input beat had to be shifted in time to align with the template. This time shift was used to correct the QRS fiducial.

Time domain HRV analysis, standard deviation of normal to normal intervals (SDNN), was performed using the Matlab implementation of the HRVAS package [5]. The simulated ECG and 12-lead Holter ECG were processed differently because each database was used for a different purpose. The simulated ECG allowed exact RR interval errors to be calculated because the exact R-wave peaks were an output of the simulator. The ESRD database was used to show effect of RR interval recovery on HRV often characterized as abnormal.

For the ESRD Holter database, pre-processing of the $\mathrm{NN}$ interval series included removing intervals which were $20 \%$ different from the running mean RR interval. SDNN was calculated on overlapping windows of length 20 minutes at an interval of 10 minutes. In the SDNN comparison for the Holter database, the SDNN reference was calculated from the RR intervals calculated from peak detection in the original 1000sps ECG.

Two aspects of high resolution RR interval recovery were tested, the impact of additive noise on the RR interval error and the impact on time domain SDNN HRV calculation of the test cases versus the reference SDNN calculation. The test case characteristics are enumerated in Table 1 below. There are two factors represented in the tests cases, RR interval correction method and the type of peak picking.

Table 1. Test case characteristics

\begin{tabular}{ccc}
\hline $\begin{array}{c}\text { Test } \\
\text { case }\end{array}$ & RR recovery method & Input ECG \\
\hline A & R-peak & Peak pick \\
B & Up-sample, R-peak & Peak pick \\
C & Up-sample, R-peak & Peak pick w/Ф \\
D & Up-sample, templ. match & Peak pick \\
E & Up-sample, templ. match & Peak pick w/ $/ \Phi$ \\
\hline
\end{tabular}

\section{Results}

The noise immunity and accuracy of the up-sample and template match methods (D \& E) can be seen in Figure 1. Method A, using R-wave peaks and no up-sampling, has the highest level of RR error standard deviation. Methods $\mathrm{D}$ and $\mathrm{E}$ have the lowest absolute RR error and little impact due to additive noise seen along the $\mathrm{X}$-axis. Methods $\mathrm{B}$ and $\mathrm{C}$ use R-wave peaks instead of template matching and the impact of noise on RR error is clear. The RR error increases with increasing additive noise.

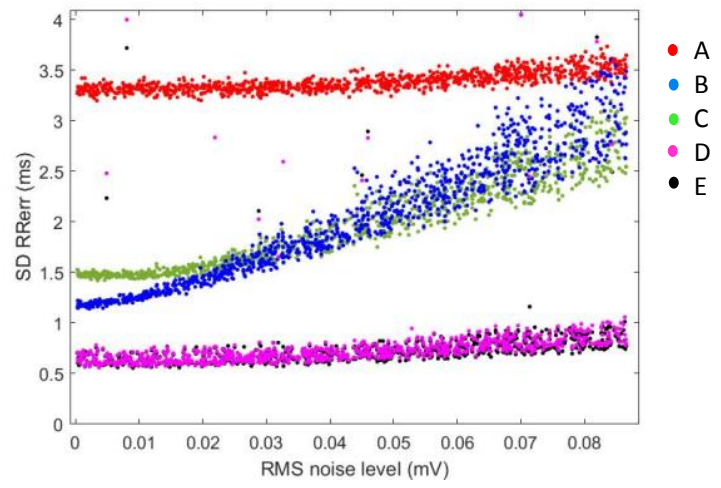

Figure 1. Scatter plot of standard deviation of RR interval error (y-axis) versus root-mean-square (RMS) additive noise level ( $\mathrm{x}$-axis) for five test cases marked A through $\mathrm{E}$ in Table 1.

Bland-Altman plots, average SDNN on the $\mathrm{x}$-axis and SDNN difference on the $y$-axis, for test cases A through $\mathrm{E}$ versus the reference can be seen in Figure 2 for the simulated ECG and 12-lead Holter ECG. Table 2 quantifies the average result seen in the Bland-Altman scatter plots for the Holter ECG.

From the left panels of Figure 2, simulated ECG, the SDNN bias at low SDNN is highest for test case A, less for test cases $\mathrm{B} / \mathrm{C}$ and lowest for test cases $\mathrm{D} / \mathrm{E}$ corresponding to $\mathrm{RR}$ recovery using template matching. The highest bias of $0.53 \mathrm{~ms}$ for test case A corresponds to a $5 \%$ error in SDNN. The bias at higher SDNN levels has less impact since the SDNN values are much higher, i.e. a $0.2 \mathrm{~ms}$ bias 
for SDNN of 60ms is very small.

The SDNN bias for the ESRD 12-lead Holter dataset was less pronounced compared to the simulated ECG database, but the effect is apparent in the summary result of Table 2. The SDNN error at low SDNN range is near zero for the template match test cases D \& E. For test case A, the bias is highest at the low range of SDNN similar to the results for the simulated ECG database. Results for test cases B \& $\mathrm{C}$ are in between.

Table 2. SDNN error (ms), recovered RR intervals versus reference RR intervals of the Holter ESRD database

\begin{tabular}{cccccc}
\hline Test & \multicolumn{5}{c}{ Reference SDNN (ms) } \\
case & 15 & 25 & 45 & 65 & 95 \\
\hline A & 3.2 & 3.0 & 3.1 & 1.8 & 1.6 \\
B & 2.1 & 2.3 & 2.5 & 1.5 & 1.2 \\
C & 2.1 & 2.4 & 2.5 & 1.5 & 1.2 \\
D & -0.3 & -0.2 & 0.5 & 1.2 & 0.8 \\
E & -0.2 & -0.1 & 0.7 & 1.3 & 0.8 \\
\hline
\end{tabular}

\section{Discussion}

Recommended RR interval time resolution for HRV analysis is based on an adequate sample rate or a lesser sample rate with interpolation to bring back the time resolution. In the case of peak picked ECG however, interpolation will not provide perfect reconstruction to correct R-wave (or S-wave) peaks. Interpolation typically is used locally just in the small number of points around the R-wave peak. As we have shown in this study, upsampling and template matching over the entire QRS duration to align beats in time reduces the RR interval error compared to just interpolation (up-sampling) around the Rwave peaks and basing RR intervals on the R-wave peaks. The difference with the algorithm presented here is that more samples are used in the correction of the rough QRS fiducial points from the QRS detector. In addition, using more points in the RR interval correction provides enhanced noise immunity as seen in the relationship between RR interval error and noise level in Figure 1.

ESRD patients generally show abnormal HRV values. For SDNN, the abnormal range is low values of SDNN. We found an erroneous increase in SDNN due to the effect of RR interval error from low sample rate and use of just $\mathrm{R}$-wave peaks to define RR intervals. The SDNN error, an increase, comes from random RR interval error which is a high frequency component of the variation of the RR interval time series. Our result is similar to the conclusion of Merri who found that this random RR error due to low sample rate manifested as a high frequency component disturbing the high frequency (HF) power and LF to HF ratio [6].

The left panels of Figure 2, the Bland-Altman SDNN scatter plot for simulated ECG, shows an abrupt increase in outliers at high levels of SDNN. This is due to a peculiar behavior of the ECG simulator at higher settings of heart rate standard deviation. For very short RR intervals, the QRS amplitude and width are both reduced to the point where the beat does not match an existing template or it matches poorly. The RR interval correction ability is lost when the beat does not match any beat-templates. On the other hand, this is a desirable behavior for the real application because a poor match means the interval is no longer normal-to-normal.

\section{Conclusion}

High resolution RR interval recovery using up-sampling and template matching resulted in reduced RR interval error and lower SDNN error than interpolation and peak detection, even when the low sample rate ECG was peakpicked. The improvements were clear for simulated ECG but also apparent for 12-lead Holter ECG from ESRD patients for whom time domain HRV is abnormally low

\section{References}

[1] Malik M, Bigger JT, Camm AJ, Kleiger RE, Malliani A, Moss AJ, et al. Heart rate variability: Standards of measurement, physiological interpretation, and clinical use, European Heart Journal, Volume 17, Issue. EuroHeartJ. 1996; 17(3): p. 354-381.

[2] McSharry PE, Clifford GD, Tarassenko L, Smith L. A dynamical model for generating synthetic electrocardiogram signals. IEEE Transactions on Biomedical Engineering. 2003; 50(3): p. 289-294.

[3] Couderc JP. The telemetric and Holter ECG warehouse initiative (THEW): a data repository for the design, implementation and validation of ECGrelated technologies. In IEEE Engineering in Medicine and Biology; 2010. p. 6252-6255.

[4] Mueller WC. Arrhythmia detection program for an ambulatory ECG monitor. Biomedical Sciences Instrumentation. 1978; 14: p. 81-85.

[5] Ramshur J. Design, Evaluation, and Application of Heart Rate Variability Analysis Software (HRVAS). PhD Thesis. The University of Memphis; 2010.

[6] Merri M, Farden DC, Mottley JG, Titlebaum EL. Sampling frequency of the electrocardiogram for spectral analysis of the heart rate variability. IEEE Transactions on Biomedical Engineering. 1990; 37(1): p. 99-106. 

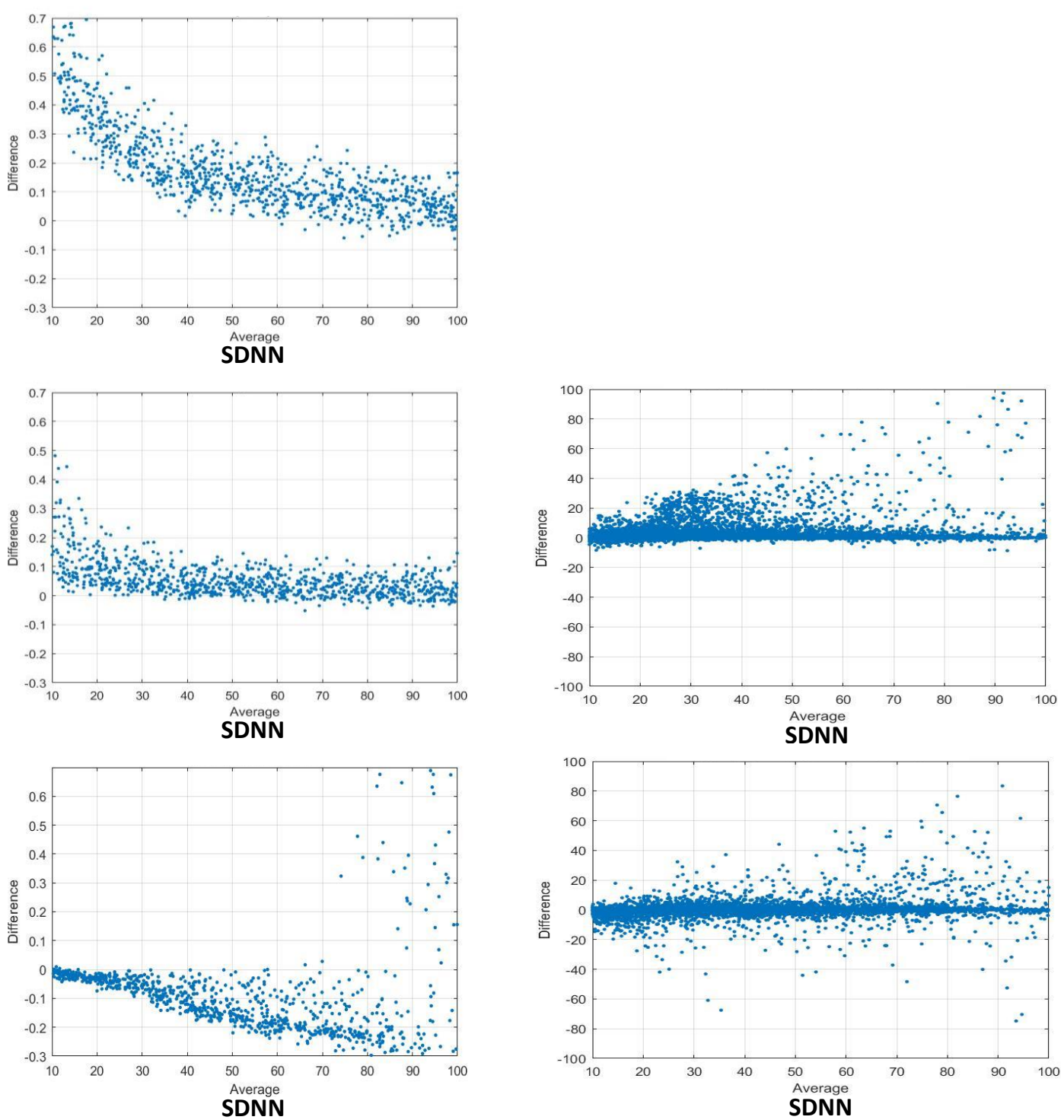

Figure 2. Bland-Altman plots of 20 min SDNN calculated from low resolution simulated ECG versus reference SDNN. Upper, middle and lower left panels show test cases A, B and D described in Table 1. Results for the Holter ESRD database are on the right. Middle and lower right plots correspond to test cases $\mathrm{C}$ and $\mathrm{E}$ from Table 1.

Address for correspondence.

Richard E. Gregg

3000 Minuteman Road, MS4201

Andover, MA 01810 USA

rich.gregg@philips.com 